\title{
FERMILAB-SLIDES-20-018-DI-LDRD-TD
}

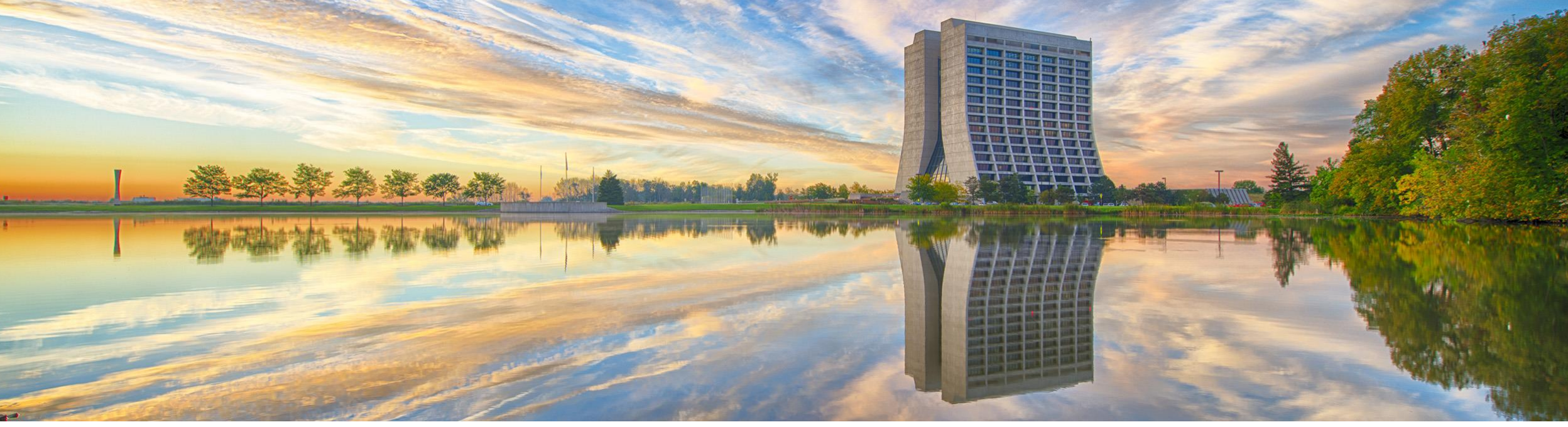

\section{Cryocooler conduction-cooled SRF cavities for particle accelerators}

Ram C. Dhuley

Cockcroft Institute Seminar, 08 September 2020

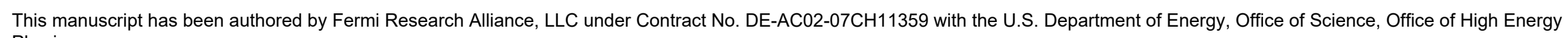
Physics. 


\section{Superconducting radiofrequency (SRF) technology has revolutionized particle accelerators for science}
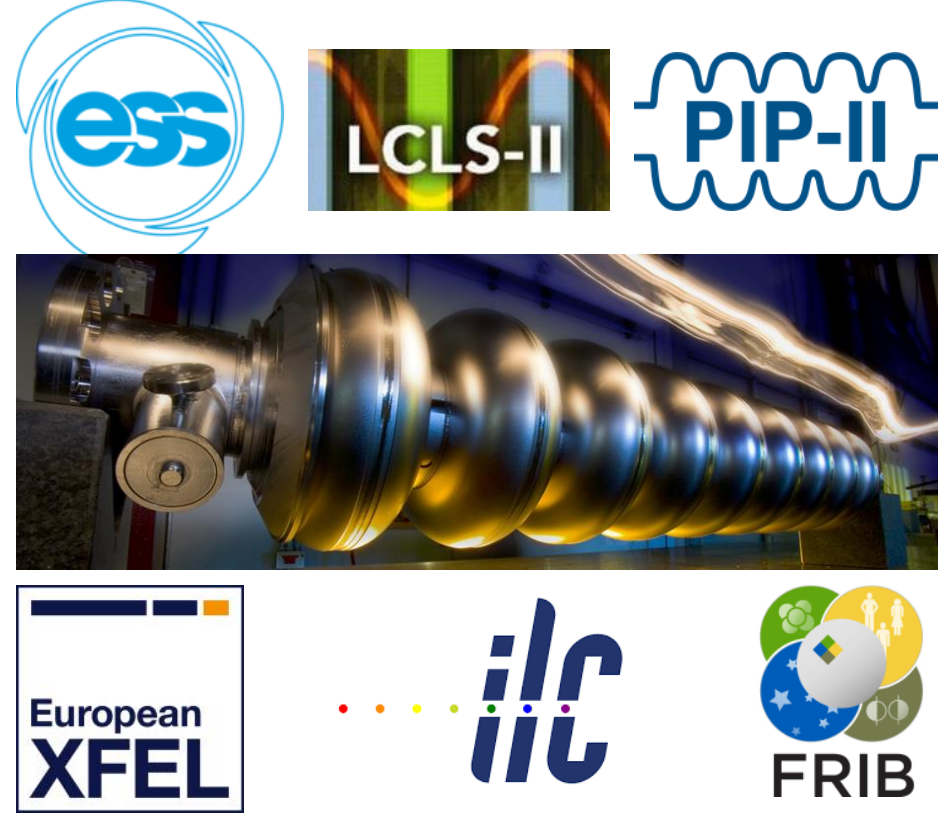

SRF benefits for large scientific machines

$>$ High wall-plug efficiency

$>$ High average beam power

Breakthroughs continue to ensue

> Niobium cavities achieve $>50 \mathrm{MV} / \mathrm{m}$

in $2 \mathrm{~K}$ liquid helium

$>\mathrm{Nb}_{3} \mathrm{Sn}$ cavities attain $>20 \mathrm{MV} / \mathrm{m}$ in $4.5 \mathrm{~K}$ liquid helium 


\section{SRF for \\ basic science}

\section{SRF for}

industry \& society

$>$ SRF relevant Industrial applications of particle accelerators?

$>$ How to make SRF suitable for industrial settings? 


\section{IARC at Fermilab}

IARC's mission: Partner with industry to exploit technology developed in the pursuit of science to create the next generation of industrial accelerators, products, and new applications.

\section{Partners}

- MWRD of Greater Chicago

- US Army Corps of Engineers (ERDC)

- Northern Illinois University

- Euclid Beamlabs

- General Atomics

\section{In-house facilities}

- Several $4 \mathrm{~K}$ cryocoolers, cryogenic test stands,

\section{Contact}

Dr. Mauricio Suarez

suarez@fnal.gov

Deputy Head of Technology Development and Industry Engagements

https://iarc.fnal.gov/ $500 \mathrm{~W}$ LHe refrigerator

- LLRF system, solid state RF power source (20 kW)

- $9 \mathrm{MeV}, 1 \mathrm{~kW}$ electron accelerator (A2D2) 


\section{Outline}

$>$ Industrial applications and scope of SRF accelerators

$>$ Cryocooler conduction-cooled SRF cavities

- Development at Fermilab

- First results

- Ongoing R\&D

> Fermilab's conduction-cooled SRF accelerator program

$>$ New R\&D facilitated by cryocooler-cooled SRF

$>$ Summary and outlook 


\section{Industrial applications and scope of SRF accelerators}

\section{Electron beam radiation} processing applications

Water/sludge/medical waste decontamination

Flue gas cleanup

Medical device sterilization Strengthening of asphalt pavements Radiation processing requires: Beam energy: 0.5-10 MeV Beam power: >>100 kW Industrial settings demand: Low capital and operating expense Robust, reliable, turnkey operation http://accelconf.web.cern.ch/AccelConf/napac2016/talks/thb3io02 talk.pdf 1-meter long SRF linac (niobium or $\mathrm{Nb}_{3} \mathrm{Sn}$ cavities) operating at $10 \mathrm{MV} / \mathrm{m}$ can provide the required energy

Small SRF surface resistance enables continuous wave (cw) operation, leading to high average beam power

At present, SRF accelerators are designed to operate with complex liquid helium cryogenic systems!

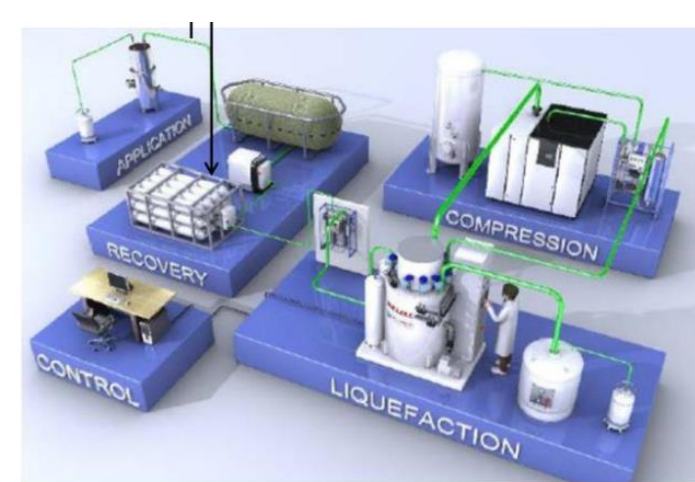

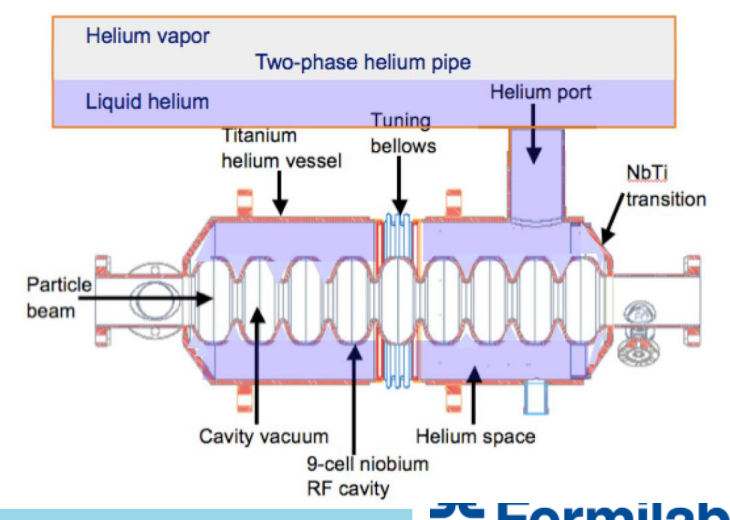




\section{Simplifying SRF cryogenics for industrial settings}

$\mathrm{Nb}_{3} \mathrm{Sn}$ cavity dissipates $~ 6-8 \mathrm{~W} @ \sim 4.5 \mathrm{~K}$ (1 m x $10 \mathrm{MV} / \mathrm{m} \mathrm{cw} ; 650 \mathrm{MHz} / 1.3 \mathrm{GHz}$ )

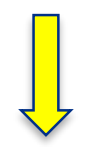

Use commercial, off-the-shelf $4 \mathrm{~K}$ cryocoolers (helium plant not required)

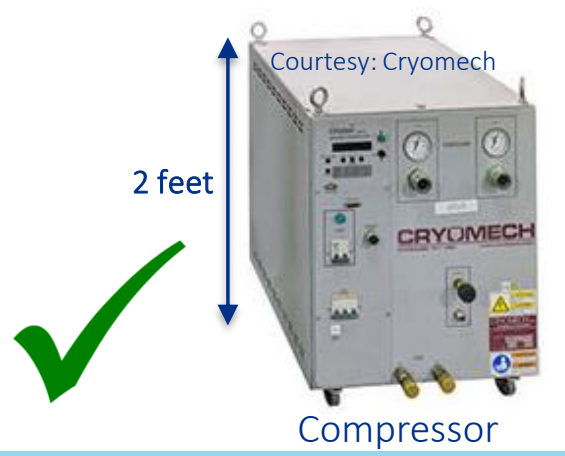

$9 / 8 / 2020$

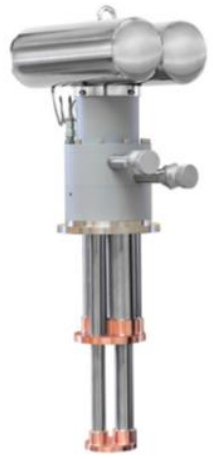

Cryocooler

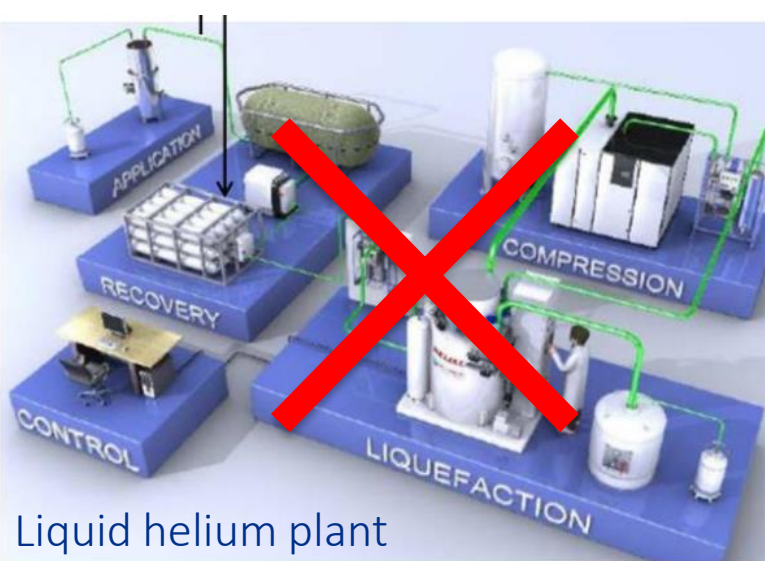

Cryocoolers offer

- Closed cycle cooling at $\sim 45 \mathrm{~K}$ and $\sim 4 \mathrm{~K}$

- Compact, small footprint

- Reliability (MTBM > 2 years non-stop operation)

- Turnkey operation (no trained operator needed, turn ON/OFF with push of a button) 


\section{Simplifying SRF cryogenics for industrial settings}

Remove cavity dissipation with thermal conduction (conduction cooling) (conventional liquid helium bath not required)
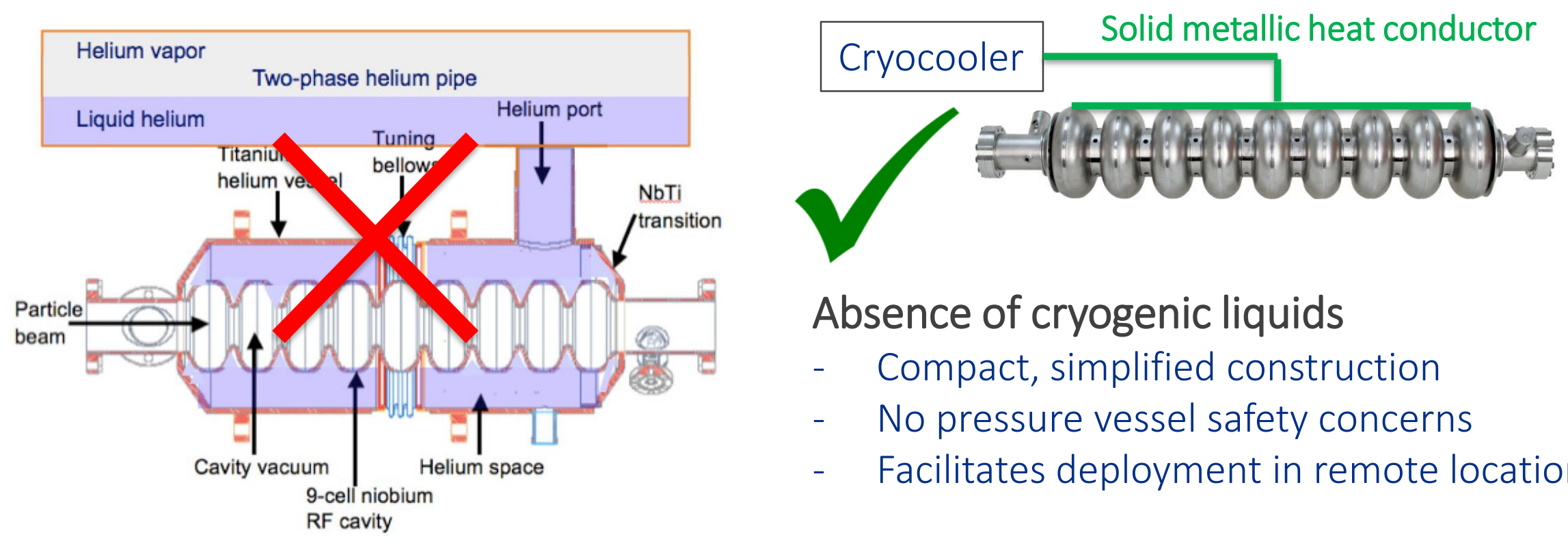

Absence of cryogenic liquids

- Compact, simplified construction

- No pressure vessel safety concerns

- Facilitates deployment in remote locations 


\section{Concept of a cryocooler conduction-cooled SRF accelerator}

R.D. Kephart, SRF2015, 2015. https://accelconf.web.cern.ch/srf2015/papers/frba03.pdf Patents: US10390419B2, US10070509B2, US9642239B2

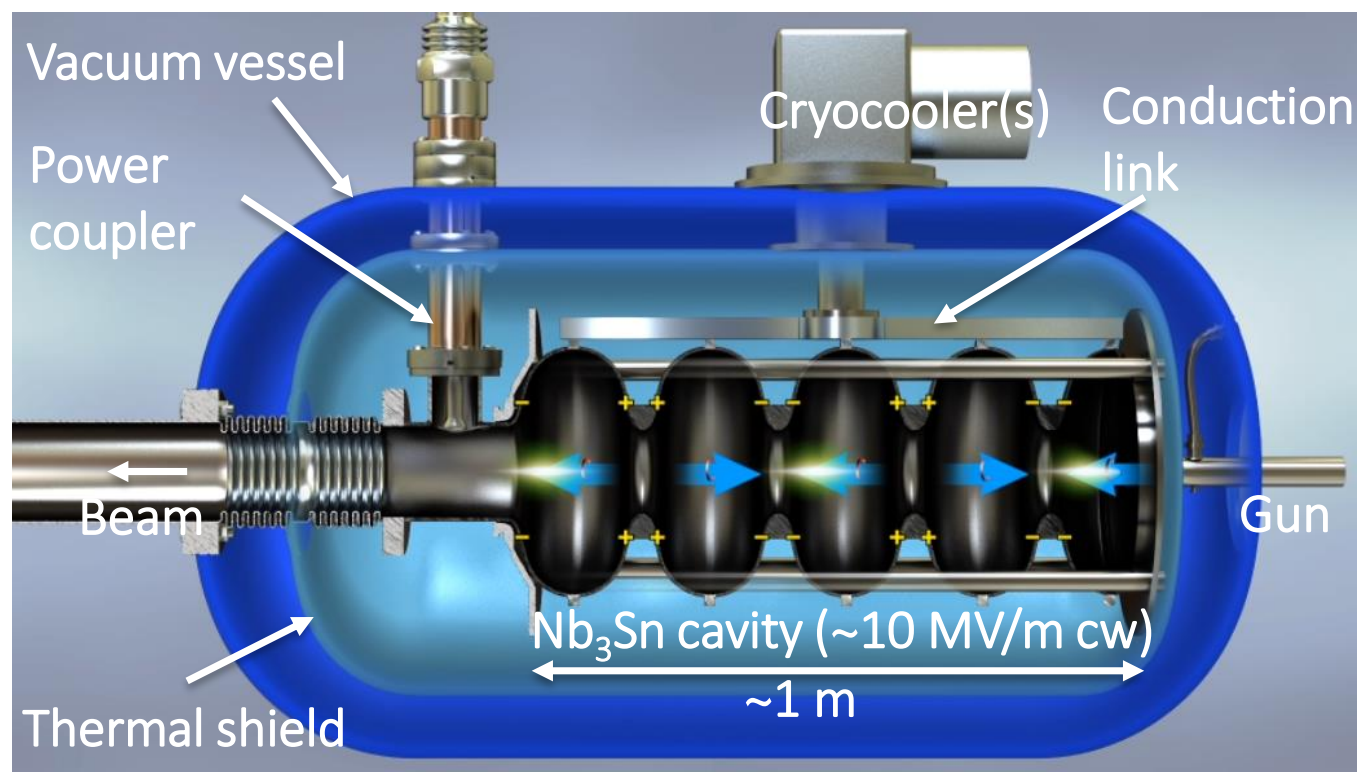

All cryogenics integrated into the module

- Cryocooler $4 \mathrm{~K}$ stage cools the SRF cavity

- Cryocooler $45 \mathrm{~K}$ stage cools thermal shield/intercept

- Enclosed in a simple vacuum vessel 


\section{Conduction-cooled SRF cavity development at Fermilab}




\section{Goal: To demonstrate $10 \mathrm{MV} / \mathrm{m} \mathrm{cw}$ on an SRF cavity with cryocooler conduction-cooling}

Our choices:

> Single cell $650 \mathrm{MHz}, \mathrm{Nb}_{3} \mathrm{Sn}$ coated niobium cavity

$>$ Cryomech PT420 cryocooler (2 W@4.2 K with 55 W @ 45 K)

$>$ High purity aluminum for the conduction cooling link

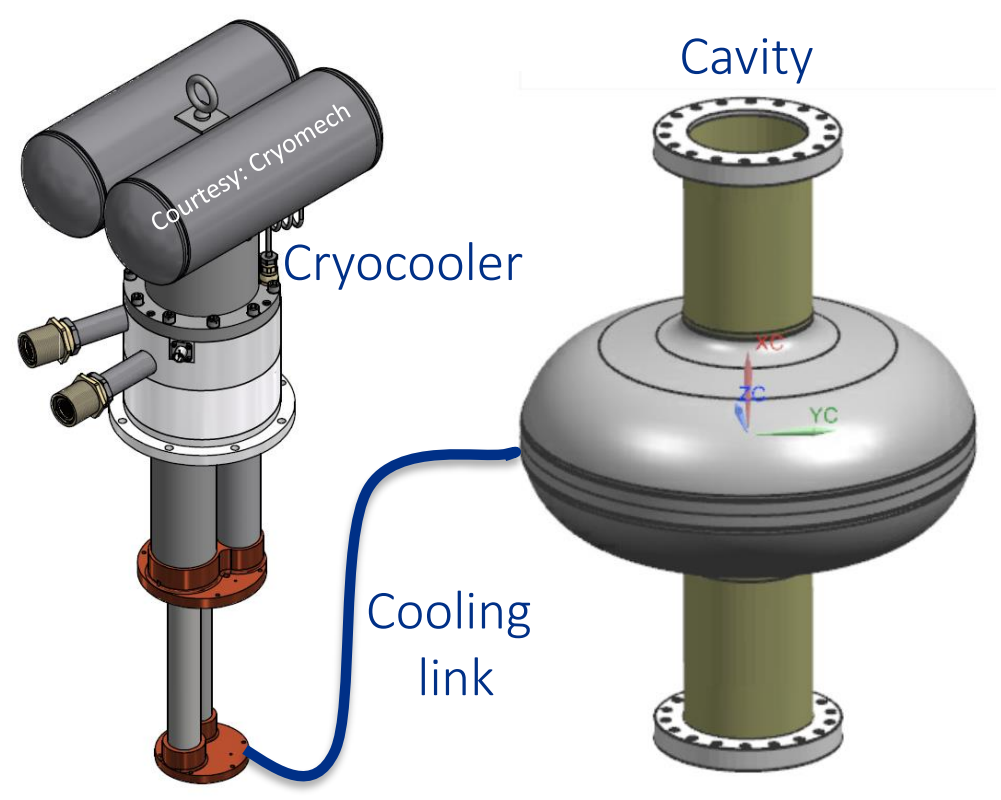




\section{Goal: To demonstrate $10 \mathrm{MV} / \mathrm{m} \mathrm{cw}$ on an SRF cavity with cryocooler conduction-cooling}

\section{Technical challenges:}

$>$ Preparing the cavity for conduction cooling

$>$ Managing thermal resistance (contact and bulk)

$>$ Magnetic shielding of the cavity

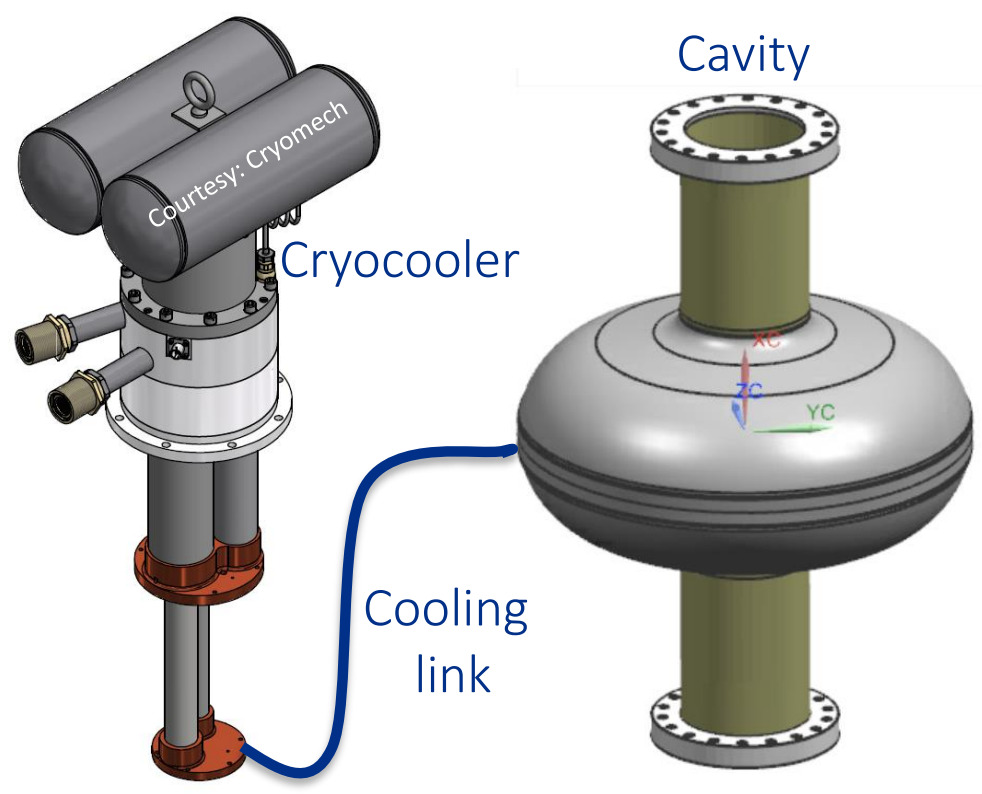




\section{Cavity preparation for thermal link attachment}

Need a thermal link attachment point on the niobium cavity shell

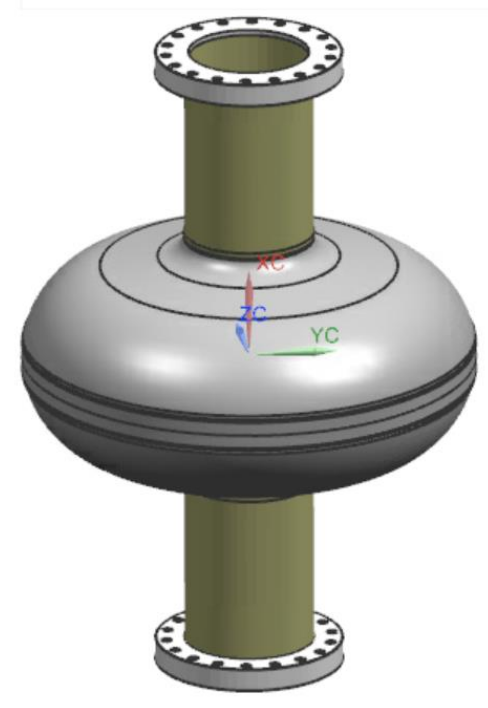

Dissipation is prominent near the equator
Solution: E-beam weld niobium cooling rings near the equator

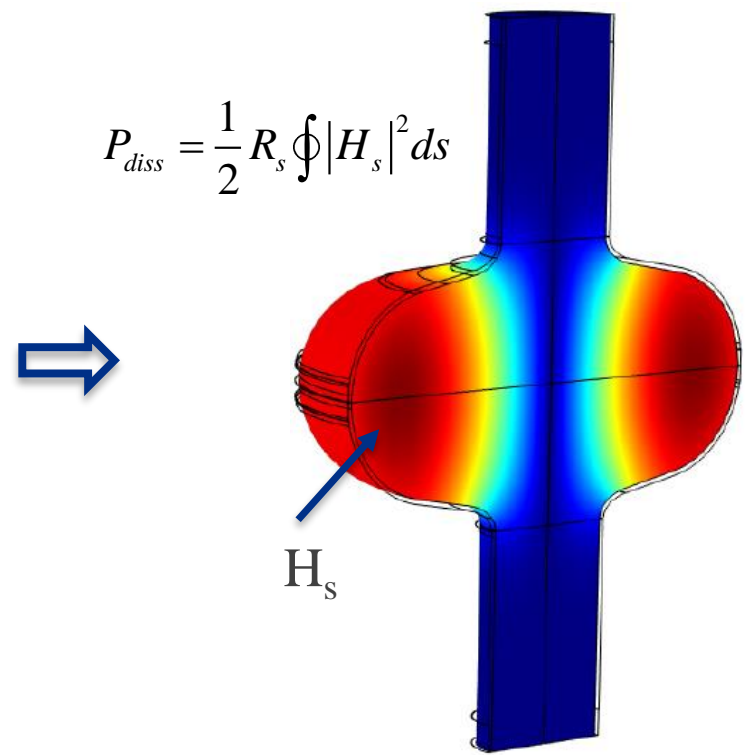




\section{Cavity preparation for thermal link attachment}

R.C. Dhuley, Provisional Patent 63/023,811

Joint design for e-beam welding

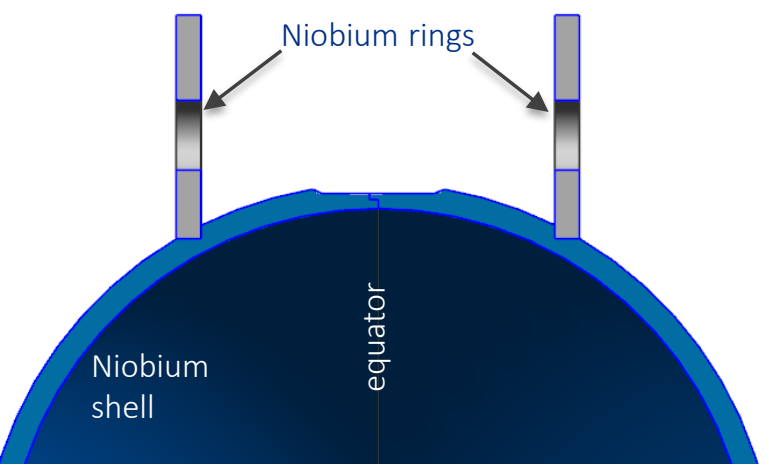

Weld development

Full penetration for thermal conductivity Avoid weld beads on the RF surface

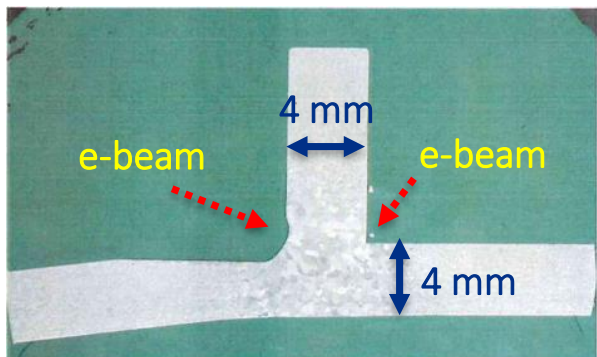

Single cell cavity ready for conduction cooling

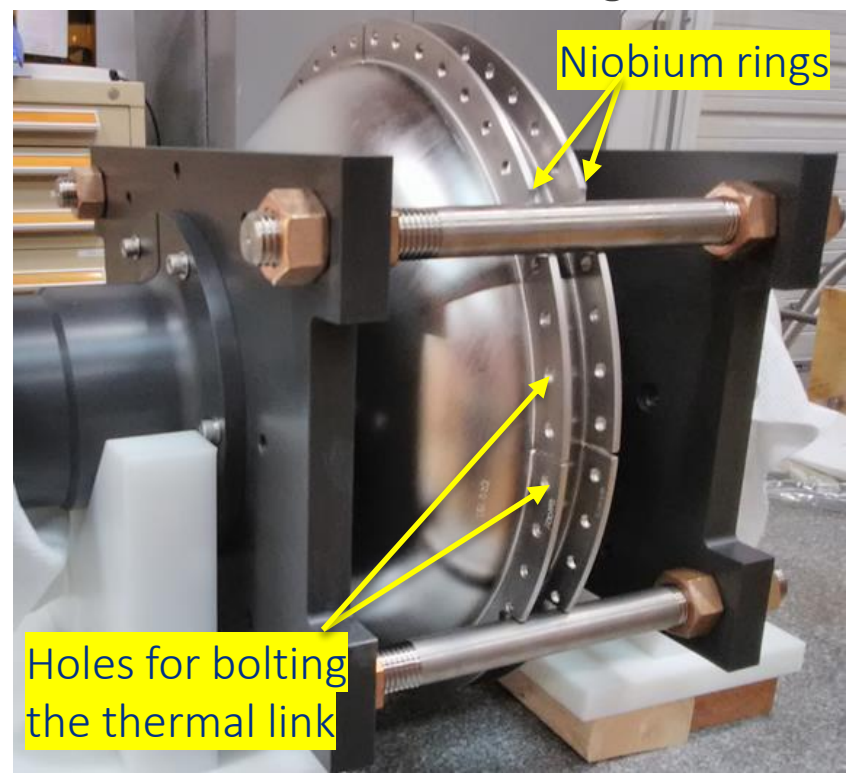




\section{Characterization of thermal resistance}

1. Cavity-link (niobium-aluminum) bolted thermal contacts

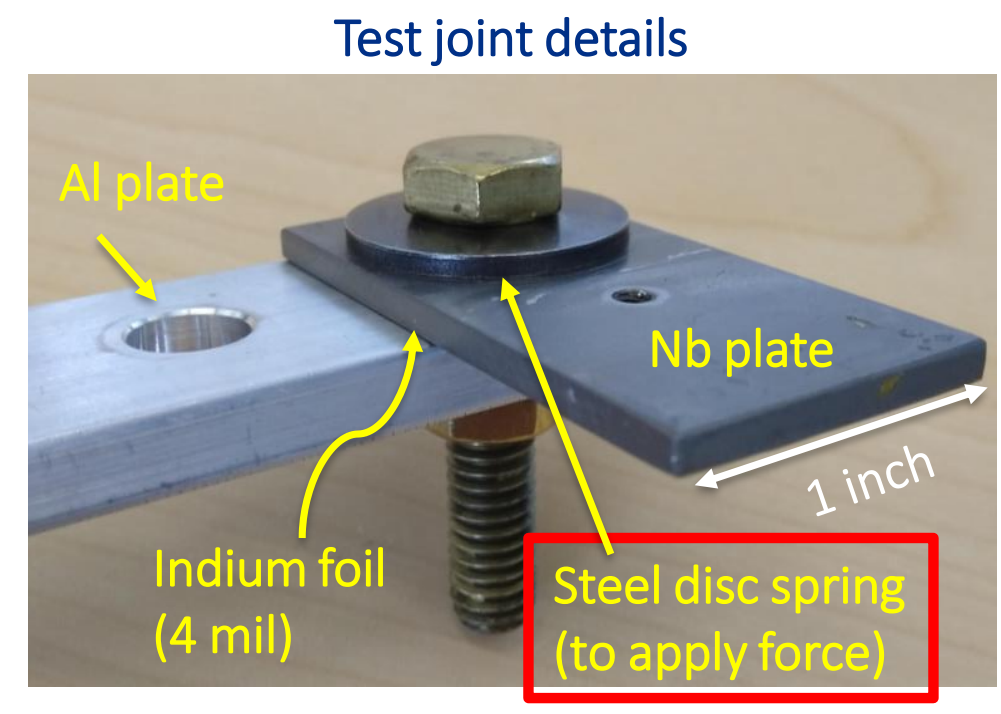

R.C. Dhuley, M.I. Geelhoed, J.C.T. Thangaraj, Cryogenics, 2018. https://doi.org/10.1016/i.cryogenics.2018.06.003

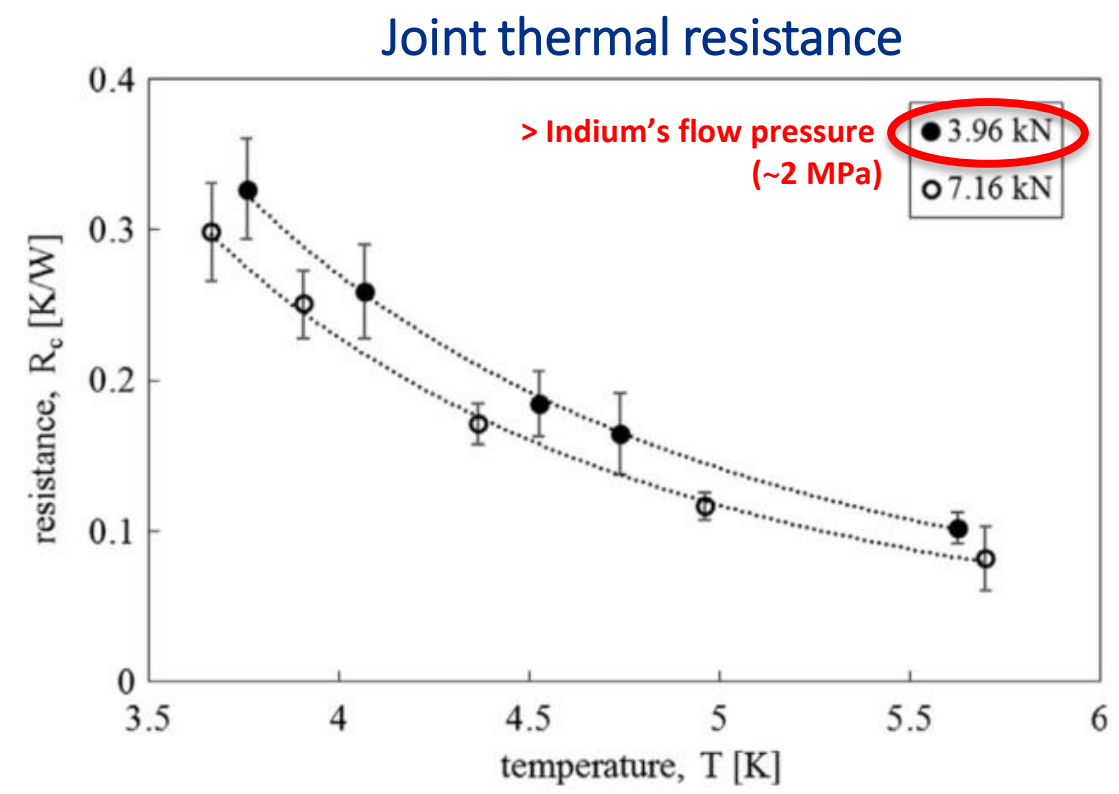

Selected design: 4 mil indium, $\sim 4 \mathrm{kN}$ force 


\section{Characterization of thermal resistance}

\section{Thermal characterization of high purity aluminum}

Setup for measuring $4 \mathrm{~K}$ thermal conductivity, contact resistance
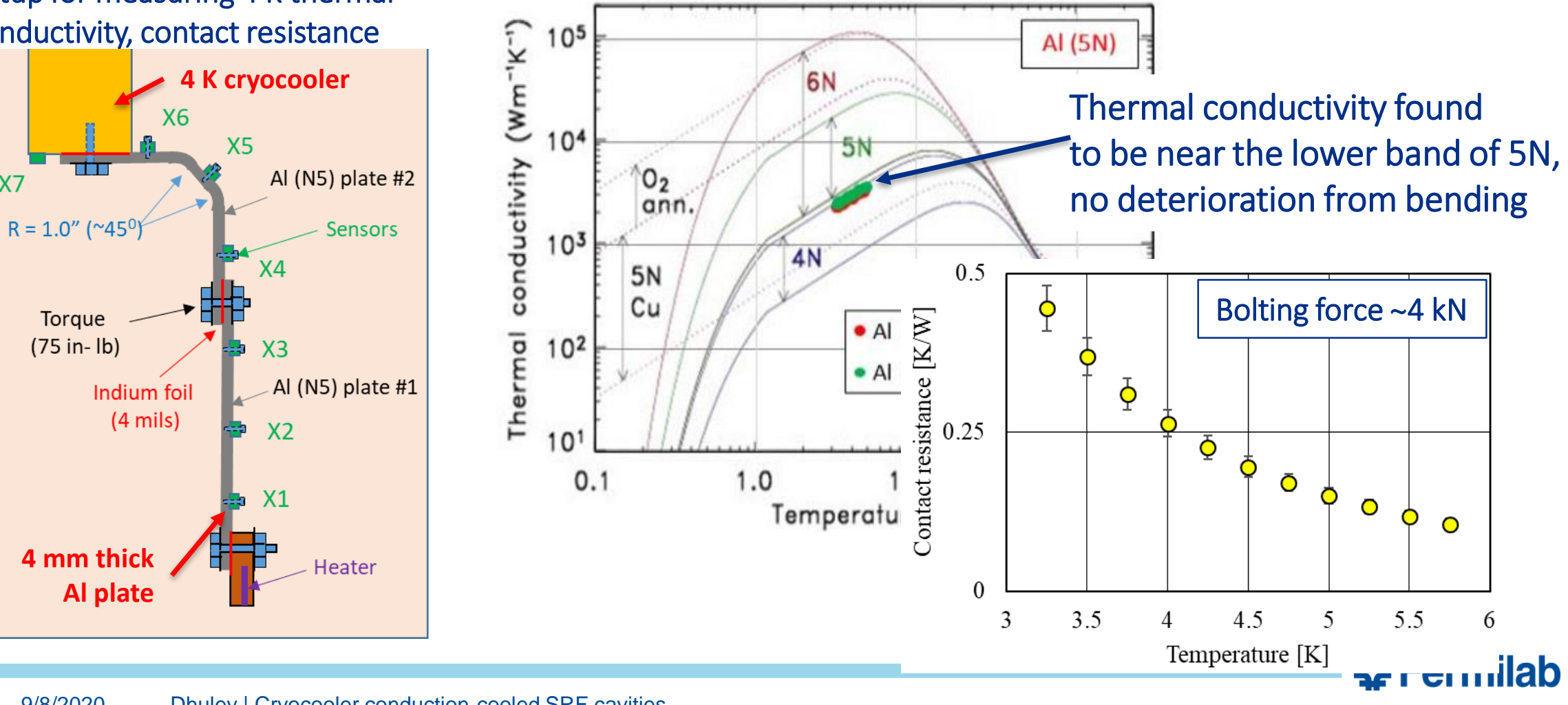


\section{Design of the conduction link design}

\section{Mechanical design; verification via multiphysics simulations}

Al conduction link bolted to the

$\mathrm{Nb}$ rings around the cavity

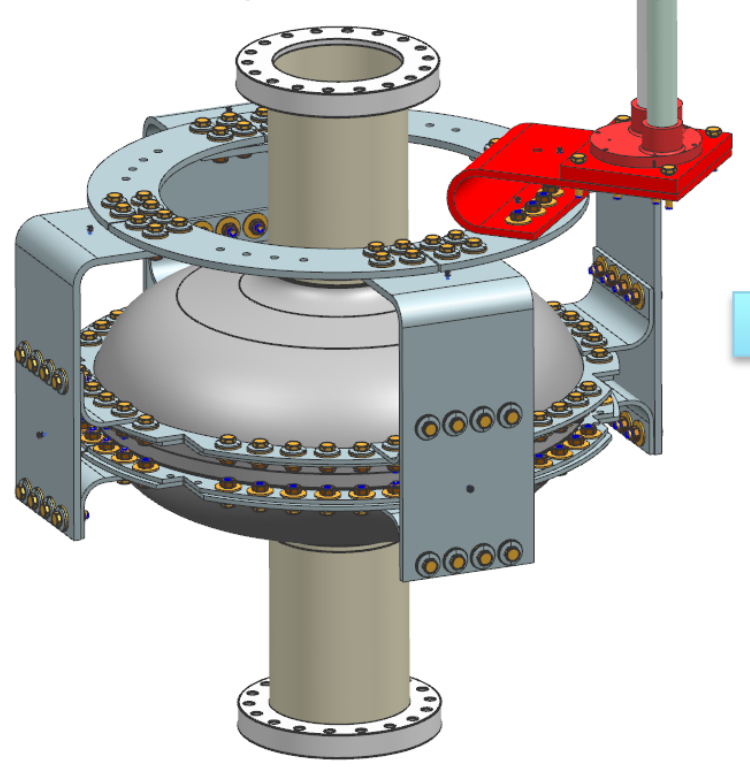

$\mathrm{Nb}_{3} \mathrm{Sn}$ surface resistance

(BCS from SRIMP + $10 \mathrm{n} \Omega$ )

$\mathrm{RF}+$ thermal simulations

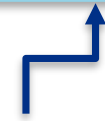

Thermal conductivities, contact resistance, cryocooler capacity
Steady state temperature profile at $10 \mathrm{MV} / \mathrm{m} \mathrm{cW}\left(\mathrm{P}_{\text {diss }} \approx 1.45 \mathrm{~W}\right) \mathrm{k}$

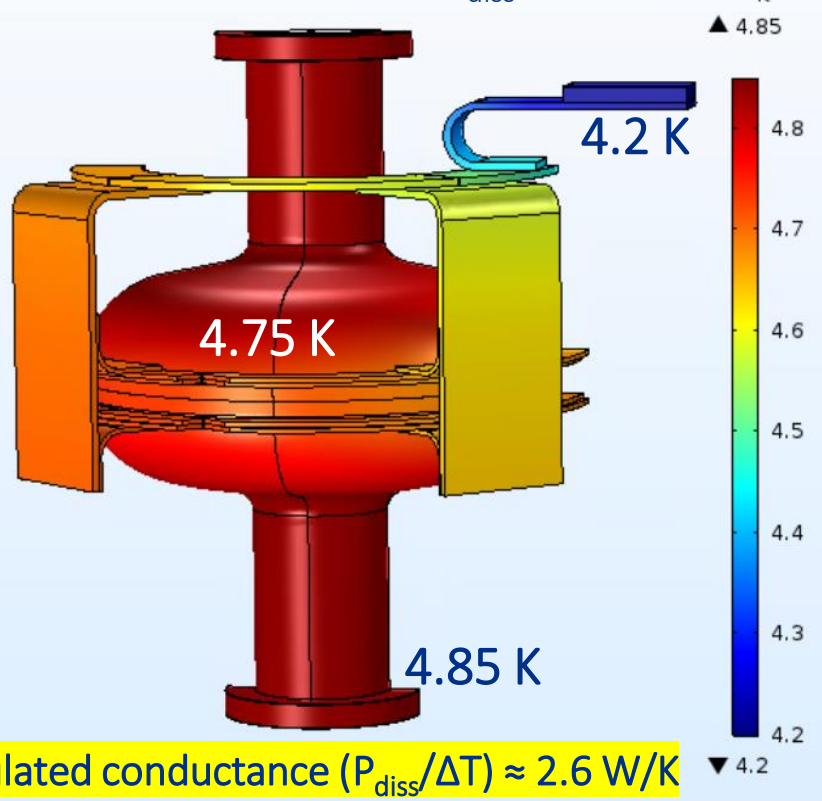

J. Thompson and R.C. Dhuley, 2019. https://doi.org/10.2172/1546003

R.C. Dhuley et al., IEEE Trans. Appl. Supercond., 2019. https://doi.org/10.1109/TASC.2019.2901252 


\section{Conduction-cooled SRF cavity measurement setup at Fermilab}

R.C. Dhuley et al., IOP Conf. Ser.: Mat. Sci. Eng., 2020. https://doi.org/10.1088/1757-899X/755/1/012136

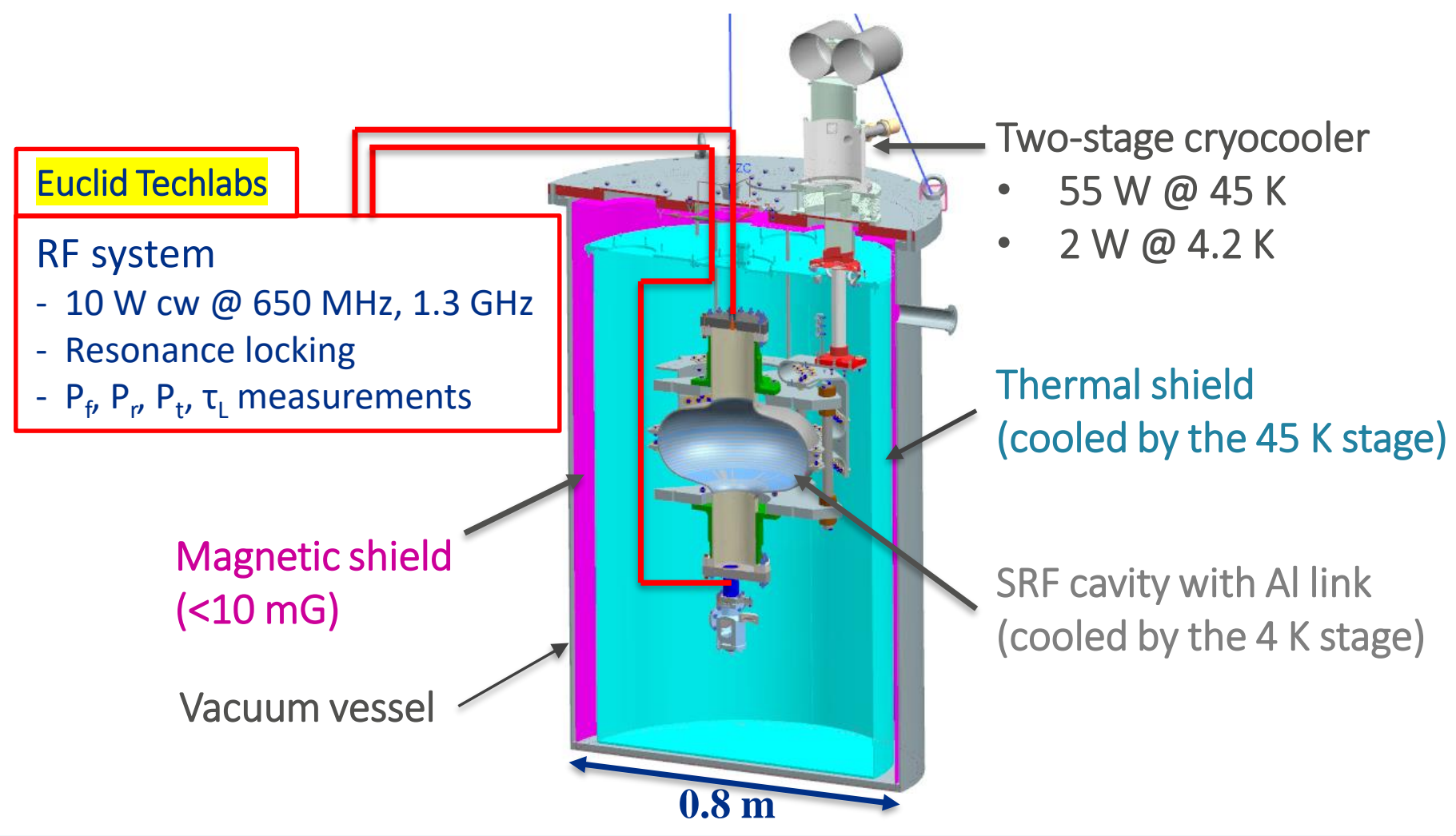


Conduction-cooled SRF cavity measurement setup at Fermilab
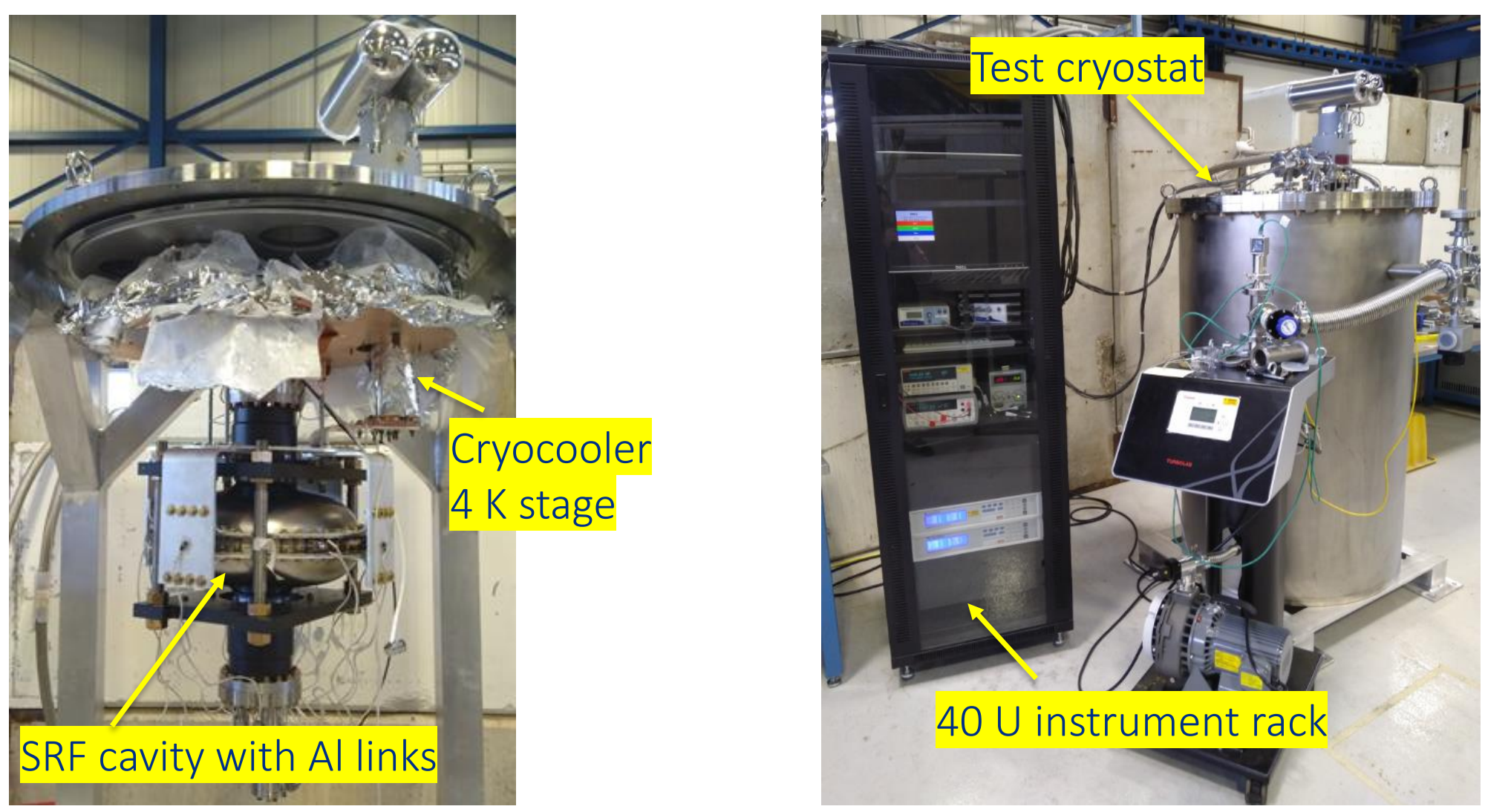


\section{Cavity processing and test sequence}

Niobium cavity with conduction rings

$\sqrt{R F}$ check, bulk EP, $800^{\circ} \mathrm{C}$ bake, light EP, HPR

$2 \mathrm{~K}$ VTS test of niobium cavity (check $10 \mathrm{MV} / \mathrm{m} \mathrm{cw}$ )

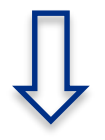

Coat with $\mathrm{Nb}_{3} \mathrm{Sn}$
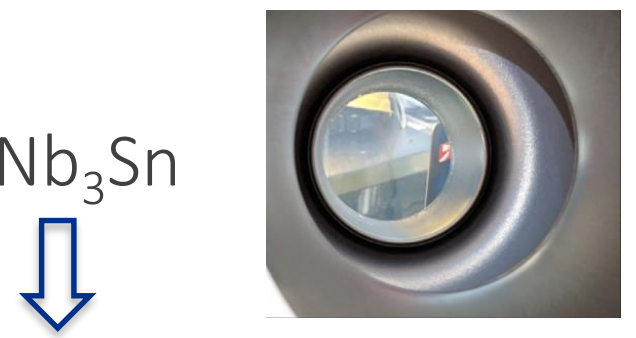

4.4 K VTS test of $\mathrm{Nb}_{3} \mathrm{Sn}$ cavity (baseline test)

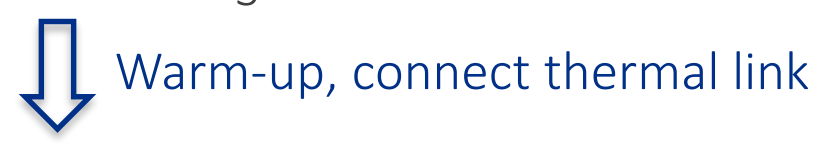

Conduction-cooled tests of $\mathrm{Nb}_{3} \mathrm{Sn}$ cavity

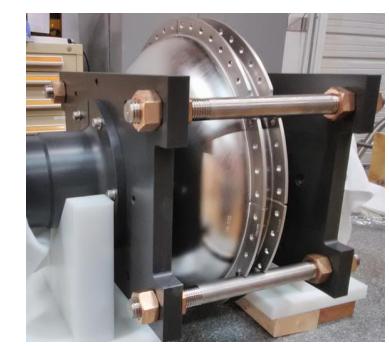

Cavity as received from vendor

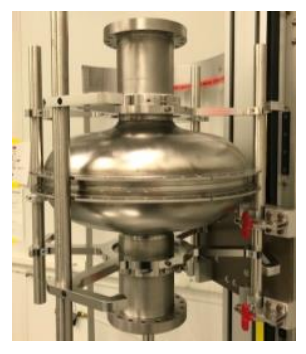

Cavity on HPR tool

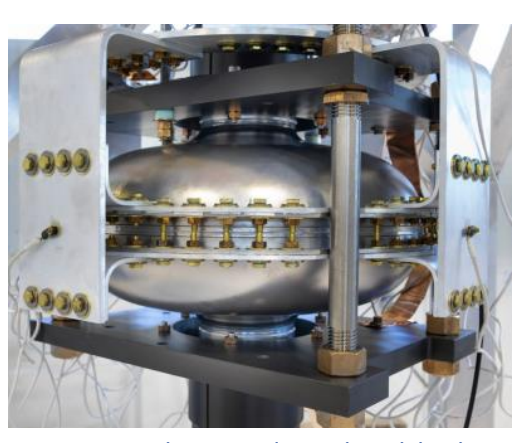

Cavity dressed with Al link 


\section{First results for the conduction-cooled $\mathrm{Nb}_{3} \mathrm{Sn}$ cavity}

R. Dhuley, S. Posen, M. Geelhoed, O. Prokofiev, J. Thangaraj, Supercond. Sci. Technol., 2020.

https://doi.org/10.1088/1361-6668/ab82f0
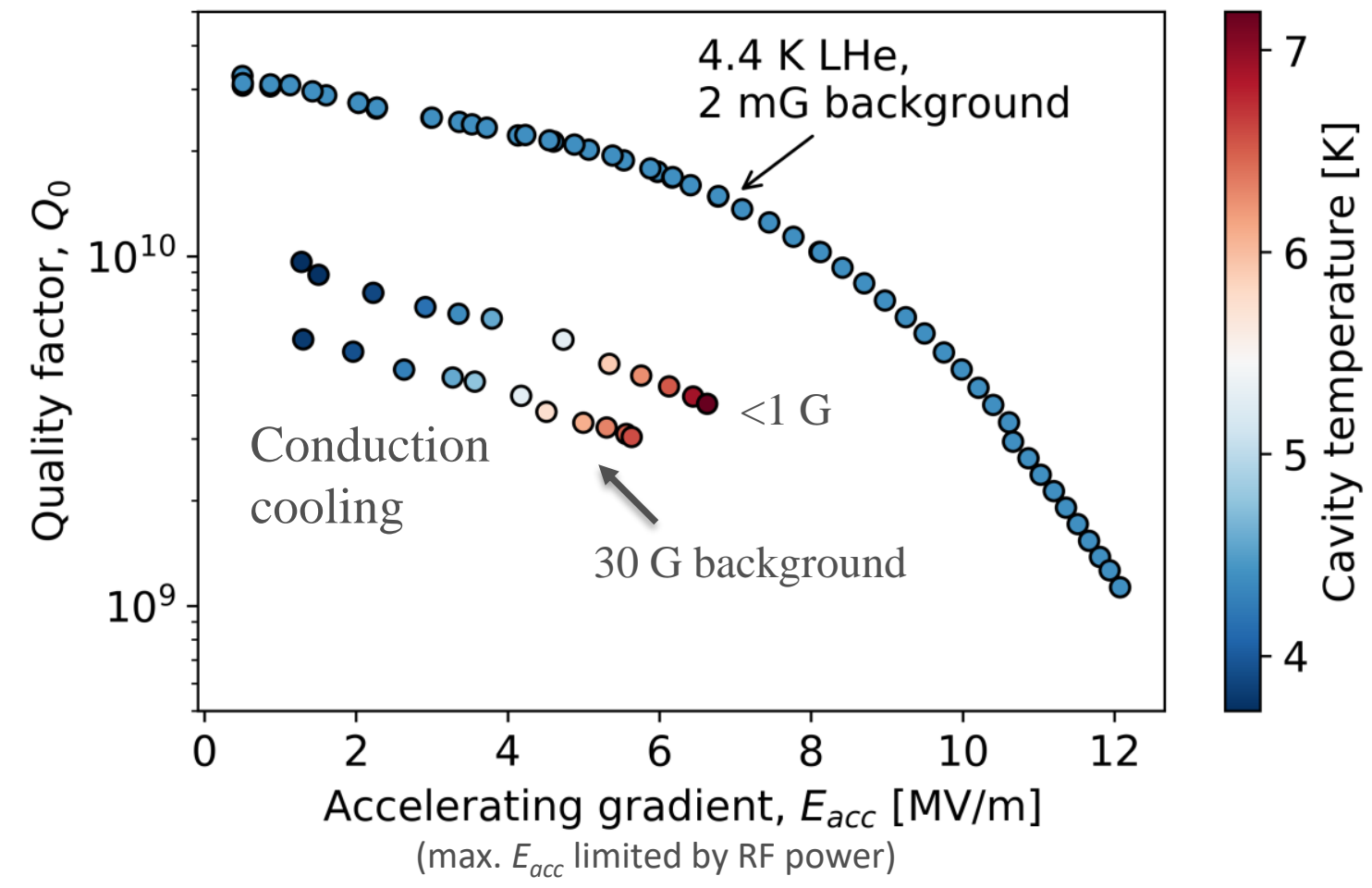

Fermilab VTS baseline with $4.5 \mathrm{~K}$ LHe

- $\quad \mathrm{Q}_{0}=3 \times 10^{10}$ at $E_{a c c}=1 \mathrm{MV} / \mathrm{m}$

- $\max E_{\text {acc }}=12 \mathrm{MV} / \mathrm{m}$

Conduction cooling

- $\quad \mathrm{Q}_{0}=5 \times 10^{9}$ at $E_{a c c}=1 \mathrm{MV} / \mathrm{m}$

- $\max E_{a c c}=5.5 \mathrm{MV} / \mathrm{m}$

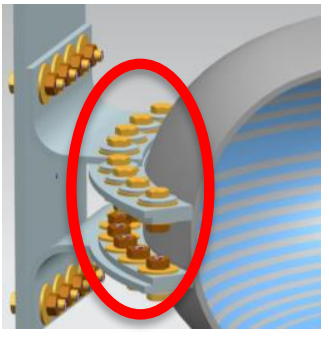

disc springs $\sim 30 \mathrm{G}$ led to large flux trapping

Conduction cooling with $<1 \mathrm{G}$ disc springs

- $\quad \mathrm{Q}_{0}=1 \times 10^{10}$ at $E_{a c c}=1 \mathrm{MV} / \mathrm{m}$

- $\quad \max E_{a c c}=6.6 \mathrm{MV} / \mathrm{m}$ 


\section{Ongoing research to reach $10 \mathrm{MV} / \mathrm{m}$}

1. Improve magnetic hygiene to reduce trapped flux
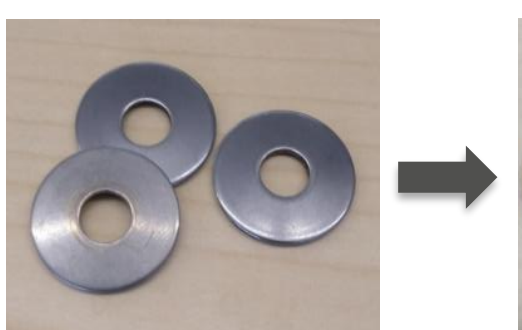

Steel (magnetic)

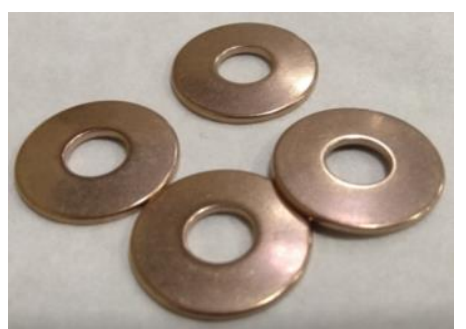

Beryllium copper (non-magnetic)

2. Flux expulsion by slow/fast cooldown using cryocooler

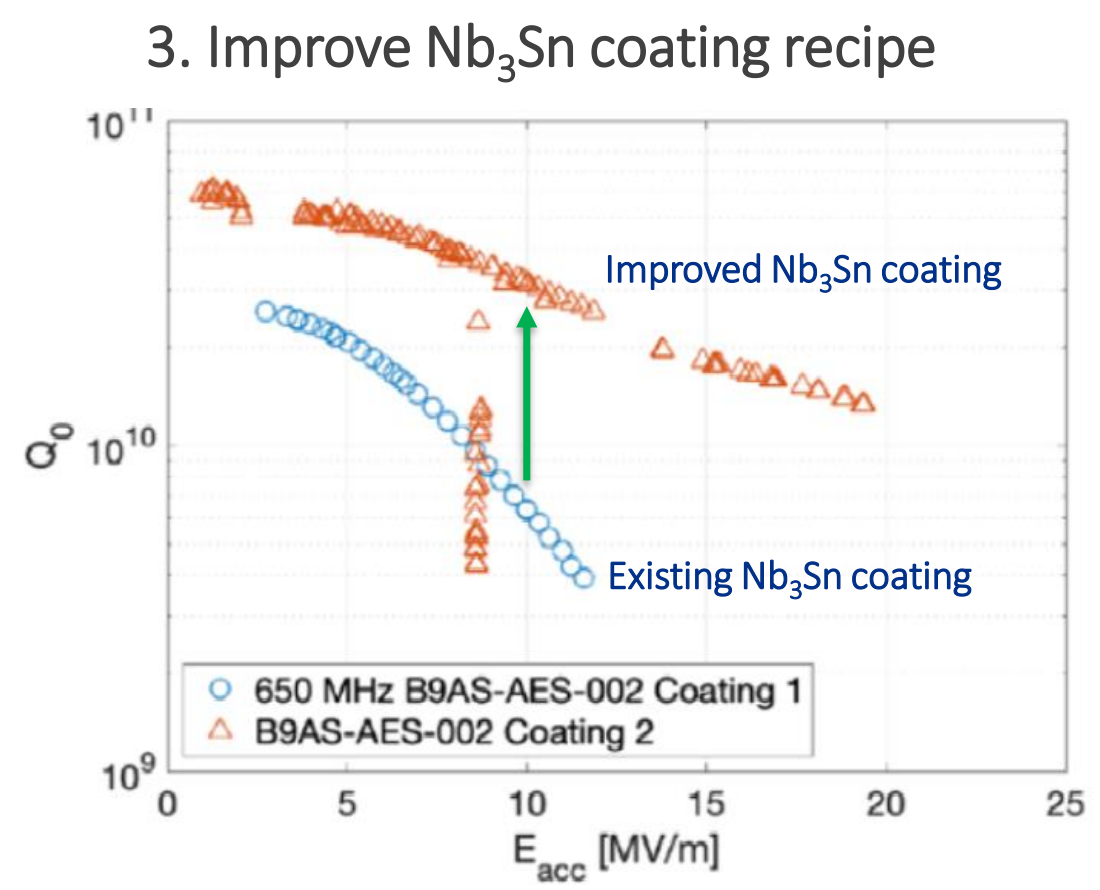

S. Posen et al., https://accelconf.web.cern.ch/srf2019/papers/thfub1.pdf 


\section{Conduction link performance, cavity thermal stability}

Comparison of measured and simulated link thermal conductance

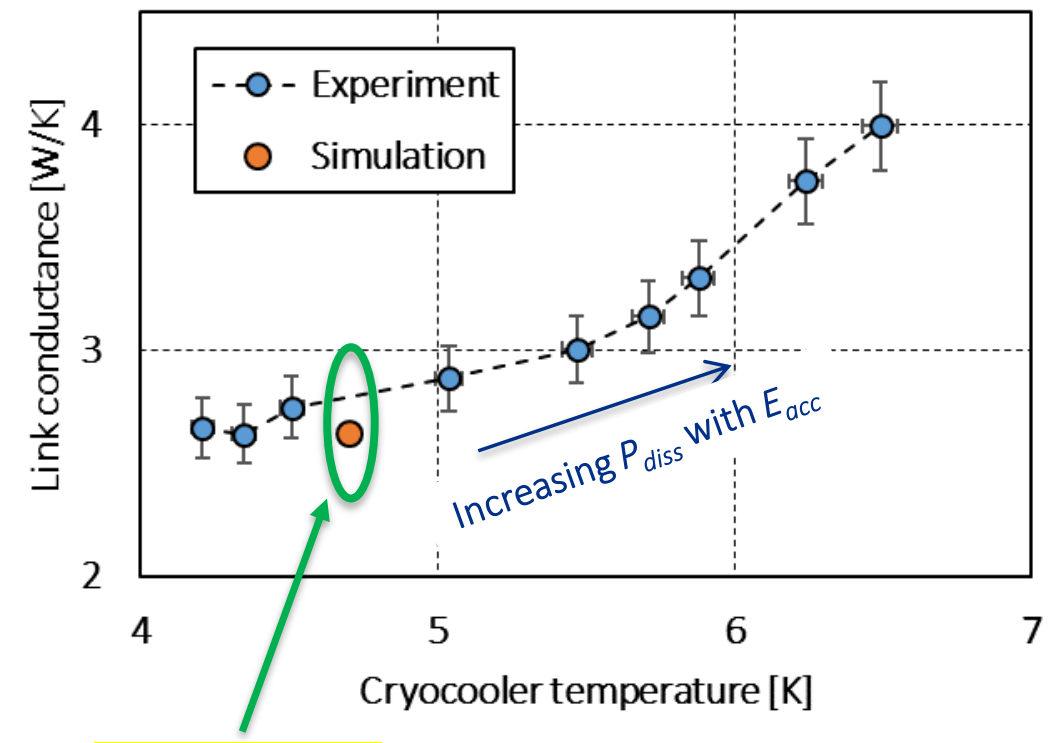

Good match!
Computed cavity surface temperature at steady state with $6.6 \mathrm{MV} / \mathrm{m} \mathrm{cw}$

- Ring temperature $=7.2 \mathrm{~K}$ (boundary condition)

- RF dissipation $=4 \mathrm{~W}$ (boundary condition)

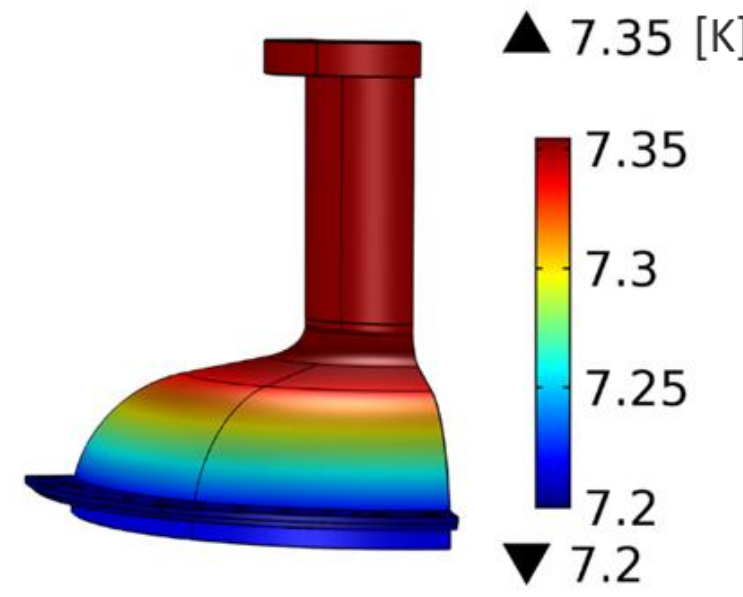

Courtesy : R. Kostin (Euclid Techlabs)
- $\Delta \mathrm{T}_{\text {spatial }}<0.15 \mathrm{~K}$

- $\mathrm{T}_{\max }<9 \mathrm{~K}$ 


\section{A new frontier in SRF is simplifying the cooling methods!}

Fermilab

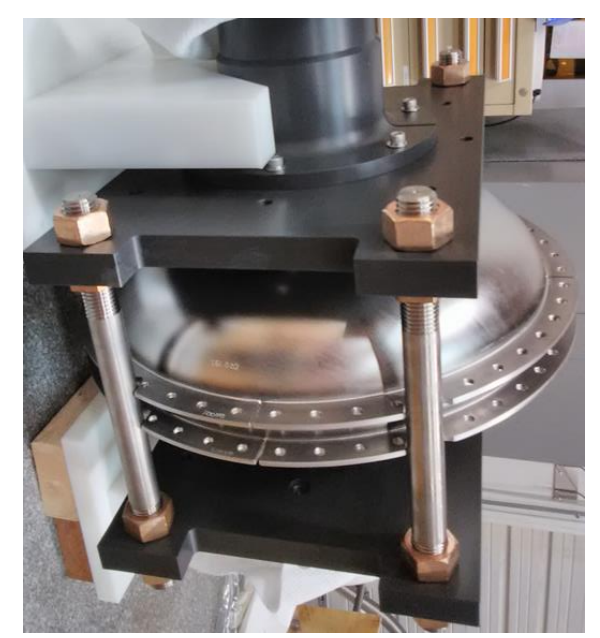

$>650 \mathrm{MHz}$

$>$ welded niobium rings
Jefferson Lab

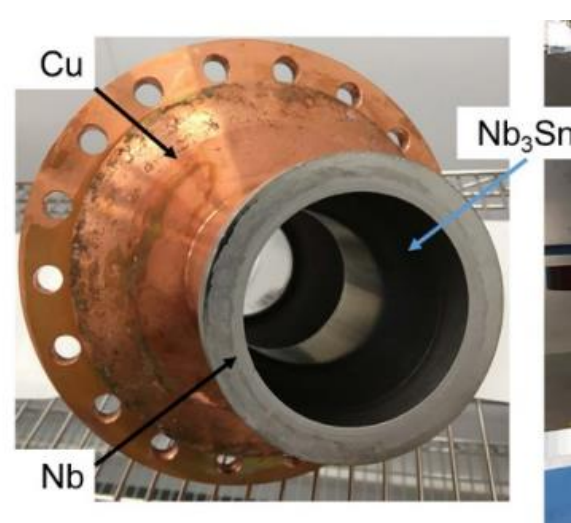

https://doi.org/10.1088/1757-899X/755/1/012136

$$
\begin{aligned}
> & 1.5 \mathrm{GHz} \\
> & \text { Cold sprayed }+ \\
& \text { electrodeposited copper }
\end{aligned}
$$

Cornell University

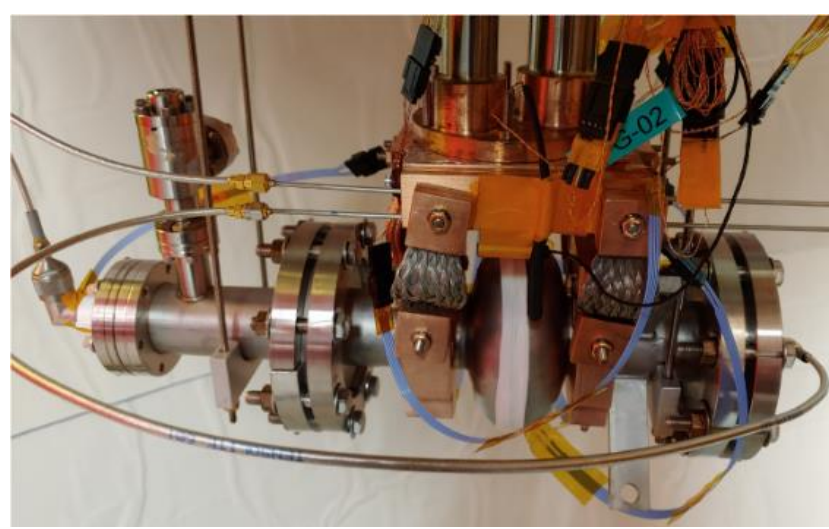

https://arxiv.org/abs/2002.11755

$$
\begin{aligned}
& >2.6 \mathrm{GHz} \\
& >\text { Copper clamps }
\end{aligned}
$$




\section{Conduction-cooled SRF accelerator program at Fermilab}




\section{Design and economics studies of industrial scale SRF electron accelerators ( $10 \mathrm{MeV},>100 \mathrm{~kW}$ )}

Supported by US Dept. of Energy HEP Accelerator Stewardship Program

\begin{tabular}{|c|c|c|}
\hline $\begin{array}{l}\text { Phase (year) / } \\
\text { Fermilab PI }\end{array}$ & Activity & Stewardship partner \\
\hline $\begin{array}{l}\text { I (2016-17) / } \\
\text { R.D. Kephart }\end{array}$ & $\begin{array}{l}\text { Conceptual design of a } 250 \mathrm{~kW} \text { and economic } \\
\text { analysis of a } 1000 \mathrm{~kW} \text { facility }\end{array}$ & \multirow{2}{*}{$\begin{array}{l}\text { MWRD of } \\
\text { Greater } \\
\text { Chicago }\end{array}$} \\
\hline $\begin{array}{l}\text { II (2017-18) / } \\
\text { J.C.T. Thangaraj }\end{array}$ & $\begin{array}{l}\text { Conceptual design of a } 1000 \mathrm{~kW} \text { module and } \\
\text { economic analysis of a } 10000 \mathrm{~kW} \text { facility }\end{array}$ & \\
\hline $\begin{array}{l}\text { III (2019-in progress) / } \\
\text { R.C. Dhuley }\end{array}$ & $\begin{array}{l}\text { Practical cryogenic design and cost analysis of } \\
\text { a } 1000 \mathrm{~kW} \text { module }\end{array}$ & \\
\hline
\end{tabular}

Design reports available at: https://iarc.fnal.gov/publications/ 


\section{New R\&D facilitated by cryocooler-cooled SRF cavities}




\section{Development of SRF based field emission sources}

\section{PI: Dr. Philippe Piot (NIU/Argonne National Lab.)}

NIU-Fermilab collaboration

- field emission cathode with nanostructured surface located in high e-field region of an SRF cavity

- use cw operation to produce high repetition rate field emission (high $I_{\text {avg }}$ )

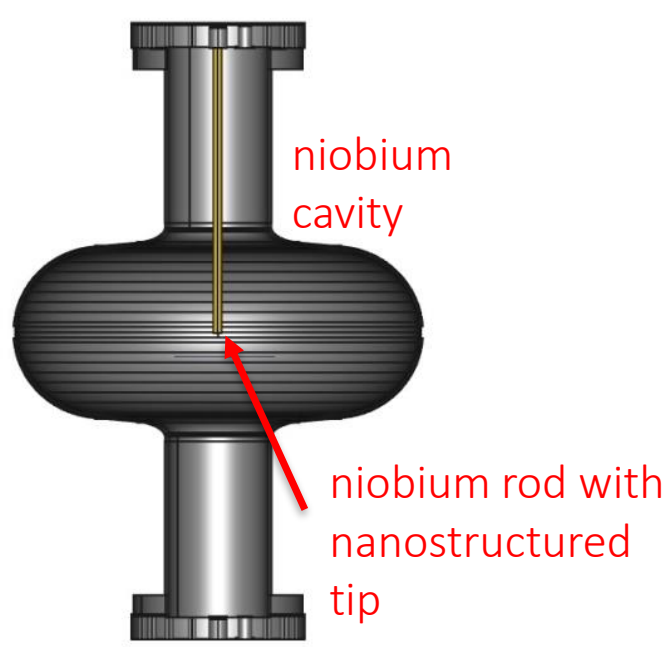

Cathode surface e-field (650 MHz cavity, $1.6 \mathrm{~W}$ cryo-cooling)

Mohsen et al., http://accelconf.web.cern.ch/ipac2019/papers/tupts083.pdf

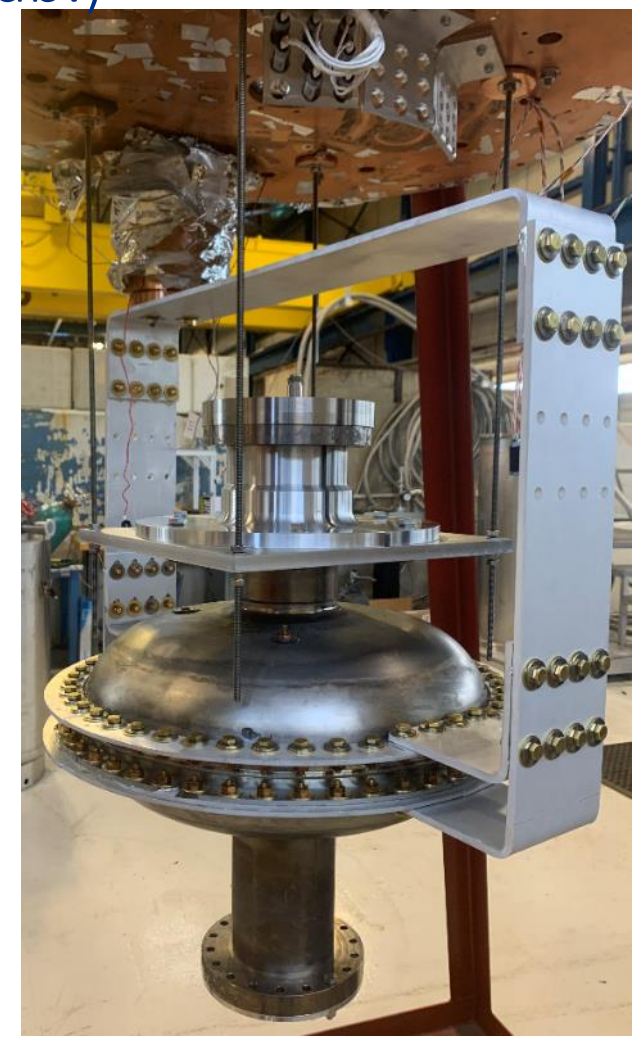




\section{Cryocooled based standalone SRF modules}

Cryocooled SRF has already been picked up by the particle accelerator industry!

S. Kutsaev et al., https://ieeexplore.ieee.org/document/9119112/

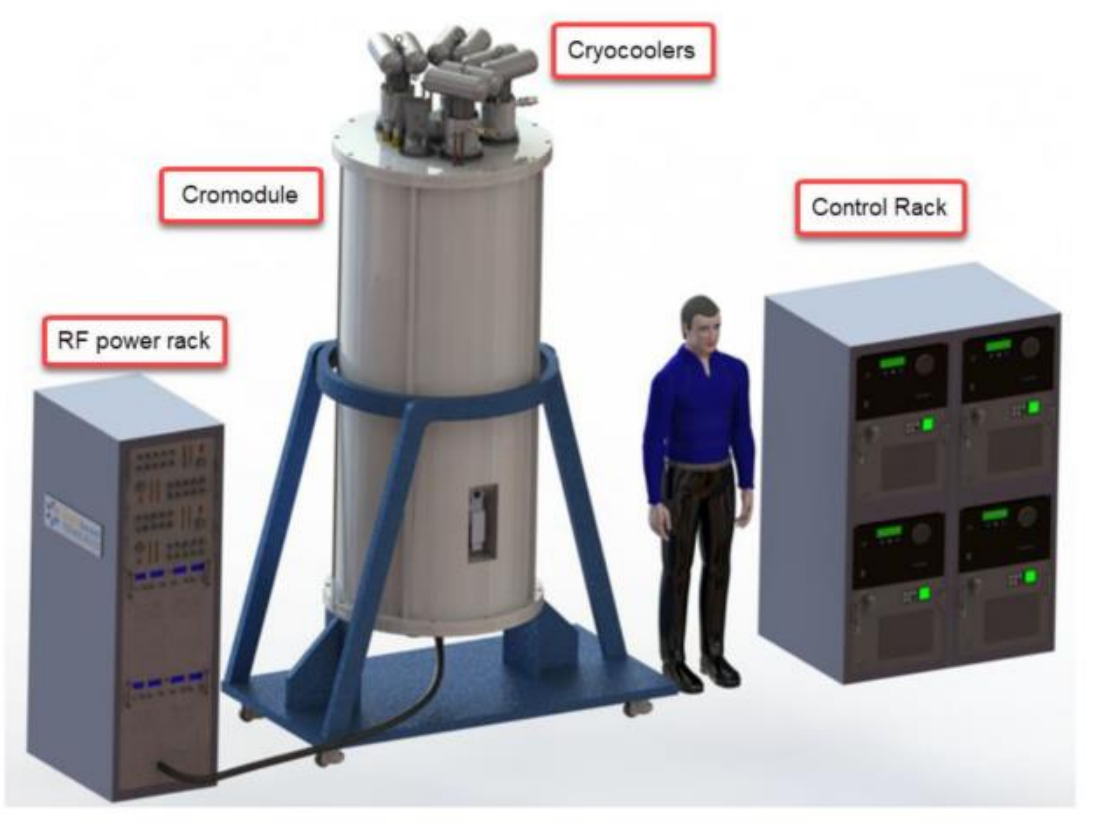

A SRF QWR cooled by pulse tube coolers for beamline upgrade at Argonne Natl. Lab.

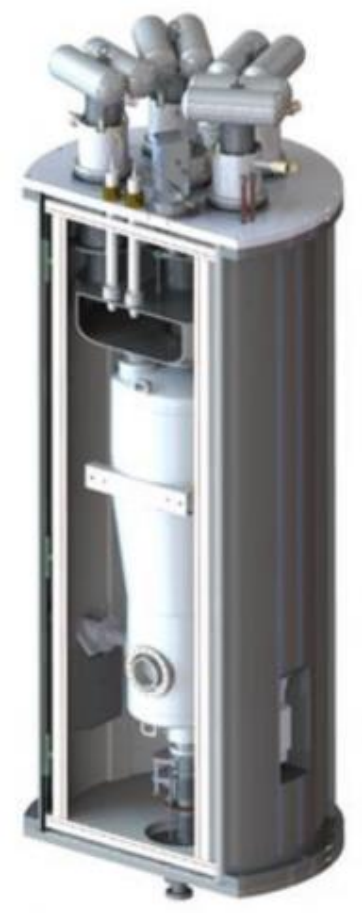




\section{Summary and outlook}

Cryocooler conduction cooling offers simple, reliable cryogenics for developing industrial SRF e-beam accelerators

Conduction-cooled SRF R\&D at Fermilab

- First demonstration $>6.5 \mathrm{MV} / \mathrm{m} \mathrm{cW}$ on a $650 \mathrm{MHz} \mathrm{Nb} 3$ Sn coated cavity

- Prototype development and high-power accelerator design in progress

Access to SRF without full stack helium cryogenic systems

- University groups, industries can embark on in-house SRF R\&D

- Standalone compact cryomodules for new SRF installations/upgrades 


\section{Acknowledgement}

This presentation has been authored by Fermi Research Alliance, LLC under Contract No. DE-AC0207CH11359 with the U.S. Department of Energy, Office of Science, Office of High Energy Physics.

- Accelerator design studies: R.C. Dhuley DOE HEP Accelerator Stewardship Award

- Conduction-cooled SRF demonstration: J.C.T. Thangaraj, Fermilab LDRD

- $\mathrm{Nb}_{3} \mathrm{Sn}$ development: S. Posen Fermilab LDRD, S. Posen DOE Early Career Award

- Compact SRF accelerator development: US Army Corps of Engineers (ERDC)

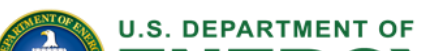 ENERCY $\begin{aligned} & \text { Office of } \\ & \text { Science }\end{aligned}$}

HEP Accelerator Stewardship

NORTHERN ILLINOIS UNIVERSITY

Northern Illinois Center for Accelerator and Detector Development

$$
\text { êuclid }
$$

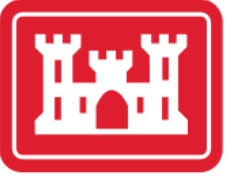

US Army Corps of Engineers (ERDC)
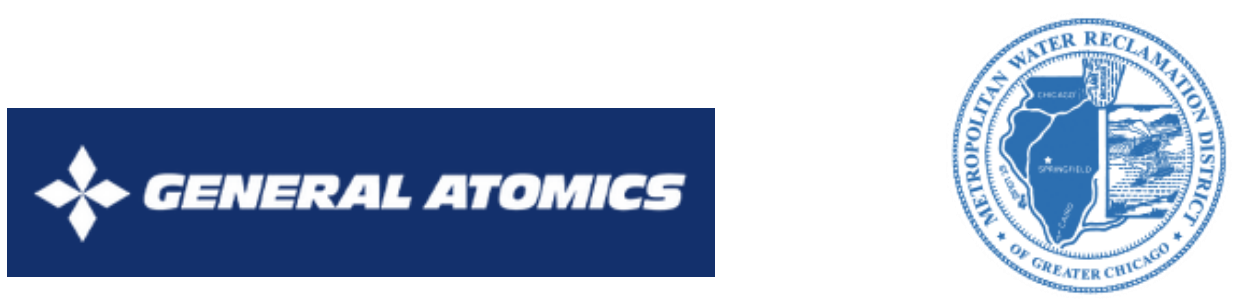